\title{
ARTÍCULOS
}

\section{EL INTERÉS POR EL CONOCIMIENTO CIENTÍFICO DE LOS ESTUDIANTES DE SECUNDARIA EN ESPAÑA*}

\author{
José Manuel Muñoz Rodríguez ${ }^{1}$ \\ María José Hernández Serrano ${ }^{1}$ \\ Sara Serrate GonzÁlez ${ }^{2}$
}

\begin{abstract}
RESUMEN: Se explora el interés de los jóvenes por el conocimiento científico-tecnológico desde tres ámbitos: la tradición familiar, los factores académicos, y el imaginario social de la profesión. A continuación se presentan los resultados de un estudio descriptivo-correlacional en el que se ha utilizado como instrumento un cuestionario para conocer los determinantes de la elección vocacional de una muestra de estudiantes de último curso de secundaria. Los resultados indican que la tradición familiar no determina ni la elección ni una visión positiva y realista de la profesión; mientras que sí es relevante la motivación del profesorado, el uso de metodologías innovadoras y el conocimiento de aspectos sociolaborales. Se concluye con la necesidad de reforzar la formación del profesorado en aspectos metodológicos y mejorar la repercusión y el valor social de la ciencia.
\end{abstract}

Palabras clave: Sociedad del conocimiento. Ciencia y tecnología. Enseńanza secundaria obligatoria. Vocación. Formación del profesorado.

\section{THE INTEREST IN SCIENTIFIC KNOWLEDGE OF SECONDARY STUDENTS IN SPAIN}

ABSTRACT: This paper explores the interest of young people in scientific
and technological knowledge, based on three areas: family tradition,
academic factors, and social imaginary of the profession. In sequence,
we present the results of a descriptive-correlational study in which a
questionnaire was used to was to determine the factors that influence
students in their final year of Secondary Education in choosing a carrier.
The results indicate that family tradition does not determine neither

\footnotetext{
*El artículo responde a los resultados del proyecto Formación en Metodologías de enseñanza activa para alumnos del máster de profesorado de secundaria de las disciplinas científico-tecnológicas. Referencia del proyecto: ID2015/0173. Universidad de Salamanca (España)

${ }^{1}$ Universidad de Salamanca, Facultad de Educación - Salamanca, España. E-mails: pepema@usal.es; mjhs@usal.es

${ }^{2}$ Universidad de Salamanca, Facultad de Ciencias Sociales - Salamanca, España. E-mail: sarasg@usal.es DOI: 10.1590/ES0101-73302019187204
} 
the choice nor the configuration of a positive and realistic view of the profession, while the motivation of teachers is relevant along with the use of innovative methodologies and the knowledge of employment aspects. Conclusions reinforce the need to strengthen teacher training in order to reformulate methodologies and improve the impact and social value of science.

Keywords: Knowledge society. Science and technology. Secondary education. Vocation. Teacher training.

\section{O INTERESSE EM CONHECIMENTO CIENTÍFICO DE ESTUDANTES SECUNDÁRIOS NA ESPANHA}

RESUMO: Este artigo explora o interesse dos jovens pelo conhecimento científico e tecnológico, baseado em três áreas: tradição familiar, fatores acadêmicos e imaginário social da profissão. Em seguida, apresentamos os resultados de um estudo descritivo-correlacional em que um questionário foi utilizado para determinar os fatores que influenciam os alunos em seu último ano do ensino médio na escolha de uma carreira. Os resultados indicam que a tradição familiar não determina nem a escolha nem a configuração de uma visão positiva e realista da profissão, ao passo que a motivação dos professores é relevante, juntamente com o uso de metodologias inovadoras e o conhecimento dos aspectos do emprego. As conclusóes reforçam a necessidade de fortalecer a formação de professores, a fim de reformular metodologias e melhorar o impacto e o valor social da ciência.

Palavras-chave: Sociedade do conhecimento. Ciência e tecnologia. Educação secundária. Vocação. Formação de professores.

\section{Planteamiento del problema}

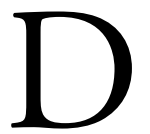

e la información, infocomunicacional, del aprendizaje, tecnológica, mediática... nos encontramos en una sociedad tildada bajo muy diferentes fórmulas (NAVAL Y ARBÚES, 2015). De todas ellas, a nuestro juicio, la que mejor aglutina las diferentes expresiones y de manera más precisa dispone el alcance y el punto de inflexión que suponen los tiempos actuales es la de Sociedad del Conocimiento, ya que es una expresión que implica apelar al conocimiento, su construcción, manejo, como eje transversal de muchos de los movimientos importantes que la sociedad crea. Teniendo en cuenta esta premisa es un hecho que las sociedades altamente desarrolladas lo serán como resultado combinado de algunos indicadores, como la difusión de las aplicaciones productivas de la ciencia y la tecnología, junto a la generación de una masa crítica de 
científicos y tecnólogos, y por supuesto la actitud proactiva que la sociedad en su conjunto mantenga ante el desarrollo científico y técnico (ALBORNOZ, 2015; ALCÍBAR, 2015).

Siendo así, quizá no seamos conscientes de que existe un problema, que hay que afrontar: el desconocimiento del conocimiento, de sus oportunidades y de sus límites (PÉREZ, 2011). Ante la pregunta a la sociedad de qué es el conocimiento, cómo se gestiona, qué es la ciencia y todo aquello que la rodea, las respuestas de jóvenes y menos jóvenes están plagadas de incertidumbres que impiden que se haga efectiva la Sociedad del Conocimiento. Un hecho que en el ámbito académico se traslada en un creciente desinterés y en la escasez de vocaciones científicas entre los jóvenes de nuestra sociedad, lo que a medio y largo plazo se traduce en una carencia de recursos humanos para hacer del conocimiento la transversalidad de la sociedad (VILACA, 2015).

Un problema que coadyuva de inmediato en un reto para las jóvenes generaciones, y para los docentes encargados de formarles: mejorar la alfabetización científica y aumentar el interés por la ciencia desde la promoción de vocaciones científicas. Hace más de una década, el Informe de la Comisión Europea sobre la Educación Científica para el futuro de Europa (EUROPEAN COMMISSION, 2007) puso de manifiesto que una de las mayores amenazas para el futuro de Europa era el alarmante descenso del interés de los jóvenes por el conocimiento científico, que podría estar llevando a la disminución del número de científicos y tecnólogos, como se ha seguido demostrando en investigaciones actuales (TASKINEN; SCHÜTTE; PRENZEL, 2013; POTVIN \& HASNI, 2014; VÁZQUEZ \& MANASSERO, 2015; ROMERO et al., 2018; HERNÁNDEZ, 2018; OSPINA, 2018). Más aún, algunos estudios sugieren que durante la educación primaria los estudiantes manifiestan una disposición positiva ante el conocimiento científico pero que este interés va disminuyendo a medida que avanza su aprendizaje, siendo la clave la transición de la etapa de educación primaria a secundaria (DEWITT; ARCHER; OSBORNE, 2014; DEWITT \& ARCHER, 2015). En este sentido, las experiencias de aprendizaje de la ciencia que se tienen a edades tempranas (entre los 10 y 14 ańos) generan una expectativa que puede desencadenar un fuerte vínculo con estas disciplinas, o por el contrario, disminuir el interés y con ello la futura atracción por la profesión (BEAL \& CROCKETT, 2010; ARCHER et al., 2012), sin olvidar el sentimiento de incapacidad que puede asociarse al estudio de disciplinas científicas y tecnológicas y que repercutiría en un desinterés y probable reactancia (BANDURA et al., 2001; SANTILLÁN et al., 2017).

Consideramos que el origen de esta problemática se sitúa en diferentes escenarios. Por un lado, la forma en que se está enseñando el saber científico y tecnológico, muy vinculado a un modelo de enseńanza predominante en la etapa de enseñanza obligatoria, dependiente de estrategias metodológicas expositivas y poco centrada en la promoción de la actividad investigadora del alumnado 
(FURTAK et al., 2012; CONTRERAS, 2016; MEDEIROS et al., 2016), lo cual reduce las experiencias de disfrute en el aprendizaje (OSBORNE, SIMON \& COLLINS, 2003; KRAPP \& PRENZEL, 2011). Por otro lado, el escaso calado social y la poca repercusión que tiene el oficio del científico, que frustra el reconocimiento de los resultados que se van produciendo (STEKOLSCHIK et al., 2010), asociado a la aún escasa divulgación que se hace de la ciencia y del conocimiento (PLAZA, 2015; KO, 2016), a pesar de los esfuerzos y nuevas fórmulas (ANGLADA \& ABADAL, 2018; BORGES; PIRES; DELGADO, 2018). Pero previo, hemos de considerar el impacto que tiene el imaginario social colectivo de la ciencia como profesión, y especialmente el que mantienen los jóvenes, que puede incidir en su decisión vocacional (ARCHER et al., 2012; DEWITT \& ARCHER, 2015) superponiéndose en ocasiones éstas percepciones globales con sus autopercepciones de competencia (BANDURA et al., 2001; JANSEN, SCHERER, \& SCHROEDERS, 2015), e incluso con las percepciones del interés que se gesta por la ciencia en su entorno familiar (TASKINEN; DIETRICH; KRACKE, 2015).

En estas tesituras, la novedad de nuestro estudio se centra en explorar en la raíz de los intereses vocacionales de los jóvenes, pues de esa manera podemos encontrar las claves para reformular las prácticas formativas y mejorar el valor social del científico. Un reto que concierne a los sectores económico, empresarial y socioeducativo, pues si estamos avanzando hacia una sociedad del conocimiento, resulta imprescindible mejorar la conexión entre los ciudadanos y el mundo de la ciencia y la tecnología. Para ellos, en esta investigación nos centramos en los estudiantes de último ańo de educación secundaria obligatoria, curso que de acuerdo con el Sistema Educativo Español deben elegir cursar algún Bachillerato relacionado, o no, con las disciplinas científicas o tecnológicas. Durante los tres cursos anteriores, además de disciplinas científicas obligatorias (Matemáticas, Biología y Geología, Física y Química) han podido cursar una materia denominada "Tecnología”, por lo que los jóvenes cuentan con un conocimiento científico-tecnológico que ha podido generarles cierto interés para proseguir cursando materias específicas en uno de los Bachilleratos que ofrece el sistema y que se denomina "Bachillerato Científico". En este sentido, buscamos la novedad y el avance del conocimiento en la percepción que el joven tiene al respecto y la manera en que determinados factores influyen en esa percepción. Para ello nos planteamos tres hipótesis, en función de los factores de estudio, que a su vez se entremezclan o superponen: la tradición familiar, los factores académicos, y el imaginario social de la profesión del científico. Si bien no podemos establecer una linealidad directa entre escoger un bachillerato y acabar desarrollando una vocación científica, o al revés, el valor de este estudio se centra en descomponer, mediante un análisis retrospectivo, los determinantes de su elección vocacional, lo que ofrece pistas para prevenir el desinterés y proyectar alternativas que contribuyan a incrementar sentimientos positivos hacia el aprendizaje de la ciencia y el fomento de vocaciones asociadas al ámbito de la ciencia y la tecnología. 


\section{Marco teórico: tres ámbitos para la contextualización del problema}

Partimos de concepto de vocación, entendido como la disposición o el interés que un ser humano tiene para dedicarse a un determinado ámbito profesional. La pregunta surge de inmediato y no es nueva: ¿qué hay de innato o de adquirido en la inclinación hacia unos estudios y profesiones u otros? La genética marca, eso es obvio, pero educativamente hemos de poner sobre la mesa la superación del mero determinismo biológico y analizar hasta qué punto las experiencias, coyunturas históricas, valores, incluso el azar, pueden marcar diferencias en la trayectoria de una persona para tomar una decisión profesional y, sobre todo, en qué ámbitos de socialización, enculturación y educación se adquieren.

La elección de una determinada especialidad, científico-tecnológica en este caso, viene construida por el conocimiento que uno va adquiriendo de sí mismo, por las representaciones sociales que le van marcando y, sobre todo, por el contexto social y académico en el que se ha ido construyendo como persona (GARCÍA et al., 2016). Un contexto que influye notoriamente, y que lo describimos desde tres ámbitos: el familiar, el académico y el social, en alusión a los valores y patrones de crianza familiar, a las formas y a los procesos de enseñanza y aprendizaje que han guiado el itinerario académico, y al imaginario y divulgación social que se ha ido construyendo y haciendo sobre su acción profesional en un momento histórico como el presente.

Con relación al primero, el entorno familiar, ha sido, y sigue siendo, el referente fundamental en la educación de una persona, precisamente cuando se moldean los intereses y apetencias profesionales. La necesidad vital del ser humano de estar en relación, y con personas adultas cuando uno es nińo, sigue situando a la familia como el núcleo principal en el desarrollo de un sujeto, contexto de aprendizaje de reglas sociales, donde el niño recibe cuidados, afectos, guías, consejos, valores, etc. Aun habiendo tenido una variación histórica en la forma de entender y vivir la institución familiar y los modelos de crianza adecuados, esta sigue siendo el primer eslabón en la construcción identitaria de un ser humano, y en la "ecología del desarrollo humano" (BRONFENBRENNER, 2005), aportando estabilidad, cuanto menos, emocional.

En este contexto, y con relación al tema que nos ocupa, algunas investigaciones indican que los intereses curriculares y las preferencias por unos $\mathrm{u}$ otros itinerarios en la enseñanza secundaria vienen determinados, de manera significativa, por el entorno familiar (KELLER \& WHISTON, 2008; MARTÍNEZ \& ZURITA, 2014). Es en la familia donde se muestran las principales pautas de socialización y aprendizaje (ALONSO \& RAMÓN, 2005; CORTÉS \& CONCHADO, 2012), lo que conlleva hablar de valores, hábitos, tradiciones profesionales, estilos y preferencias vitales que, a su vez, marcan las preferencias 
académicas y profesionales. También desde diferentes investigaciones, se ha defendido que el nivel socio-económico y cultural de la familia, la clase y el rol social que desempeñan, incide en las preferencias académicas y vocacionales (RUÍZ-CARRASCOSA \& MOLERO, 2010).

De igual forma, la colaboración de la familia con los centros educativos es un indicador de mejora en el rendimiento académico del niño y, en consecuencia, sitúa al individuo en mejores condiciones para tomar decisiones sobre su futuro académico y profesional, al sintonizarse los dos ámbitos donde por excelencia se dirimen las principales decisiones del joven (THOMAS; WHYBROW; SCHARBER, 2012).

Con relación al segundo, nadie pone en duda la gran responsabilidad que tiene el entorno académico para fomentar la vocación científica y tecnológica en sus alumnos; el espíritu emprendedor, la creatividad, la curiosidad, el afán por descubrir, entre otros elementos, fomentan una serie de competencias y destrezas básicas en los alumnos que implican el fomento de la vocación científica, y que pueden, y deben, inculcarse desde los procesos de aprendizaje.

La educación científica, entendida como el fomento de actitudes determinantes hacia la vocación científica, implica la generación de actitudes positivas y favorables hacia la ciencia y la tecnología con el objetivo de que un mayor número de alumnos se inclinen por hacer estudios propios del mundo de la ciencia y la tecnología. "El desarrollo de actitudes positivas hacia la ciencia y la tecnología ha sido un objetivo olvidado por la educación tradicional para científicos, centrada en la enseñanza de conocimientos y procesos" (VÁZQUEZ Y MANASSERO, 2009, p. 321). Si analizamos las metodologías predominantes en la enseñanza de las ciencias, los estudios insisten en que se ha abusado del lenguaje racional frente al emocional, mediado por la palabra, con escasa alusión a otro tipo de lenguajes, más cercanos al ámbito de los afectos, con los cuales hacer más atractiva la ciencia y el mundo del conocimiento (ACEVEDO-DÍAZ \& GARCÍA-CARMONA, 2016). El uso de lo cognitivo, a lo sumo lo conductual, infravalorando lo afectivo para enseñar y hacer atractiva la ciencia y la tecnología, para programar y actuar con la didáctica de las ciencias como herramienta, ha supuesto una infravaloración de lo emocional, lo que ha derivado en el cierre de puertas por parte de los alumnos a determinados campos por el exceso de raciocinio y metalismo al que ha sido sometido (PEÑA et al., 2016).

$Y$ en lo que atañe al tercer elemento indicado, el mundo de la ciencia y la tecnología hay que precisar que las investigaciones muestran cierto nivel de diferenciación entre la visión que el entorno social tiene de la ciencia respecto de la que tiene de la tecnología. A la ciencia, por un lado, se le pide que sea esclarecedora, evidente, incluso divulgativa, pero, por otro lado, siempre se le responsabiliza de la lentitud con la trabajan y el poco alcance que tiene a corto plazo. Bien es cierto, que el entorno social también se apoya en la ciencia para buscar argumentos a favor de mejorar los problemas personales y sociales que afectan a la humanidad. 
La imagen social de la ciencia y la tecnología, es positiva y ha aumentado en los últimos años (FECYT, 2015), lo cual es valorable dentro de los cánones utilitaristas, en cuestiones como la salud, el medio ambiente, el transporte y la energía, o la biotecnología, como áreas que afectan directamente a la vida de las personas.

Dentro del contexto social, un elemento significativo es el del género, y la diferenciación que existe en las vocaciones científicas en función del mismo. El entorno social, como muestran algunas investigaciones, influye sobre la vocación científica, siendo la diferenciación por género una importante variable contextual que de manera notoria mediatiza la elección de los alumnos. Los diferentes estudios de los últimos años muestran la existencia de estereotipos en las carreras y sus respectivas profesiones, aunque éstas dependan del nivel de desarrollo de los diferentes países (VÁZQUEZ \& MANASSERO, 2015).

En resumen, estos tres ámbitos de contextualización, familiar, académico y social, están en la base del problema planteado inicialmente sobre la escasez de interés por el conocimiento científico en los jóvenes. De acuerdo con el marco teórico presentado, nos planteamos tres hipótesis:

H1: La tradición familiar determina la elección de un bachillerato relacionado con las disciplinas cientifico-tecnológicas y tiene una influencia positiva en la construcción del imaginario social de la profesión.

H2: Los alumnos que han decidido estudiar un bachillerato relacionado consideran que los factores académicos (profesor, contenidos, metodología, rendimiento...) tuvieron influencia en su decisión vocacional.

H3: Los alumnos que han decidido estudiar un bachillerado relacionado consideran que los factores sociales (opinión de los adultos, conocimiento de la profesión, expectativas laborales, estereotipos de género...) tuvieron influencia en su decisión vocacional.

\section{Metodología}

Se ha seleccionado un diseño de naturaleza cuantitativa con metodología de tipo no experimental (KERLINGER Y LEE, 2002), recurriendo al método descriptivo-correlacional a través del estudio de encuestas (BERENDS, 2006). Inicialmente, se ha llevado a cabo una revisión de los instrumentos de encuesta existentes en torno al tema de investigación, a partir de los estudios de Osborne, Dillon \& College (2008), Becker (2009) y de manera especial el estudio de Polino \& Chiappe (2011) para los jóvenes iberoamericanos. A partir de esta revisión se ha diseñado el cuestionario sobre: "Interés por el conocimiento científico y tecnológico de los alumnos de educación secundaria" que se validó a través de juicio de expertos y 6 profesores de educación secundaria de disciplinas científicas; además se realizó un primer estudio piloto con 36 estudiantes de secundaria. 
A excepción del primer bloque con cuestiones demográficas (género, edad, profesión del padre/madre, modalidad de bachillerato elegido) el resto de preguntas se contestaban con una escala formato Likert de 1 a 4 puntos de menor a mayor grado de acuerdo. El bloque dos del cuestionario estaba compuesto por preguntas en torno a la imagen social de científico. El bloque tres incluía un conjunto de actividades formativas para el desarrollo de las clases de Ciencias Naturales/Tecnología, valoradas como más o menos frecuentes. Y un cuarto bloque para valorar la influencia de diversos factores en la decisión vocacional. En cuanto a la consistencia interna del instrumento se ha utilizado el coeficiente Alfa de Cronbach, para cada uno de los bloques que componen el cuestionario, cuyos resultados muestran indicios de fiabilidad, con valores superiores a .877 en todos los casos.

\section{Muestra participante}

La muestra de la investigación está conformada por estudiantes de cuarto de la ESO, pertenecientes tanto a centros públicos como concertados de la provincia de Salamanca. A través de un muestreo de tipo no probabilístico causal o incidental, la muestra definitiva quedó constituida por 280 estudiantes de cuarto curso de la ESO, de los cuales un 54,6\% $(n=153)$ son hombres y el $45,4 \%(n=127)$ son mujeres.

Para proceder a los análisis comparativos del estudio se seleccionó como variable discriminante el tipo de bachillerato elegido por los alumnos para continuar sus estudios. El $41 \%$ de la muestra decidió estudiar un bachillerato relacionado con la Ciencia/Tecnología (de los cuales, el $61 \%$ son hombres, y el $39 \%$ son mujeres), el $46 \%$ proseguirá en estudios superiores no relacionados ( $46 \%$ hombres y $54 \%$ mujeres), mientras que el $13 \%$ aún se muestra indeciso.

\section{Resultados y conclusiones}

En cuanto a la primera de las hipótesis planteadas se observó que solo el $30,7 \%$ contaba con tradición familiar (padre/madre/entorno familiar cercano son profesionales del área de la Ciencia y la Tecnología, $\mathrm{n}=86$ ). A pesar del bajo porcentaje, los datos muestran correlación entre estas dos variables $(r=0.142$, p 0.05), esto quiere decir que es ligeramente más probable $(51,2 \%)$ que quienes cuentan con dicha tradición realicen estudios superiores relacionados. Mientras que también es importante considerar que un tercio del alumnado (36,1\%), que no cuenta dicha tradición, sí manifiesta su intención de cursar estudios vinculados a la ciencia y/o tecnología.

Para analizar el impacto de la tradición familiar en el conocimiento de la profesión y en la construcción del imaginario social, se analizaron cinco aspectos vocacionales positivos (parte izquierda de la Figura 1) y cuatro negativos (parte derecha). De acuerdo con la línea horizontal que divide las puntuaciones medias 
entre el desacuerdo ( 1 y 2 ) y el acuerdo (3 y 4 ), podemos observar que, respecto a los aspectos positivos, los alumnos que han decidido cursar un bachillerato relacionado manifiestan un grado de acuerdo más elevado, existiendo diferencias estadísticamente significativas $(\mathrm{p}=0,000)$ al considerar que es una profesión útil, apasionante, de trabajo en equipo y que desarrolla la creatividad.

Respecto a los aspectos vocacionales negativos, llama la atención que mayoritariamente los alumnos, tanto los que cursen un bachillerato relacionado como los que no, estén de acuerdo en que es un trabajo al que hay que dedicar muchas horas de trabajo (94,2\% está de acuerdo o muy de acuerdo); mientras que para el resto de las cuestiones planteadas se ha detectado un elevado desacuerdo. Si bien, otra posible lectura de este desacuerdo estaría indicando una visión positiva de la profesión por parte de todo el alumnado, con independencia de la elección de Bachillerato; es decir, que un porcentaje considerable del alumnado sí considerarían que sea una profesión bien retribuida económicamente $(63,1 \%)$, con perspectivas laborales $(69,3 \%)$ de la que no se derivan consecuencias graves o efectos no deseados $(56,7 \%)$.

Respondiendo a nuestra primera hipótesis, los datos sugieren, al igual que otras investigaciones (ARCHER et al., 2012) que se está abandonando el hábito familiar de continuar con la tradición, no existiendo ya una fuerte determinación como hace unas décadas (FERRY; FOUAD; SMITH, 2000; KELLER \& WHISTON, 2008), al menos en lo que refiere al ámbito científico-tecnológico. Y además que la tradición familiar, por sí sola, no discrimina como variable de influencia en la construcción del imaginario social de la profesión del científico. Es más bien la elección vocacional, ya tomada, la que lleva a considerar la profesión desde una visión más positiva o menos.

\section{Figura 1}

Imaginario social de la profesión del científico, según la media del acuerdo (4) y desacuerdo (1) de los aspectos positivos (izquierda) y negativos (derecha). 4,00
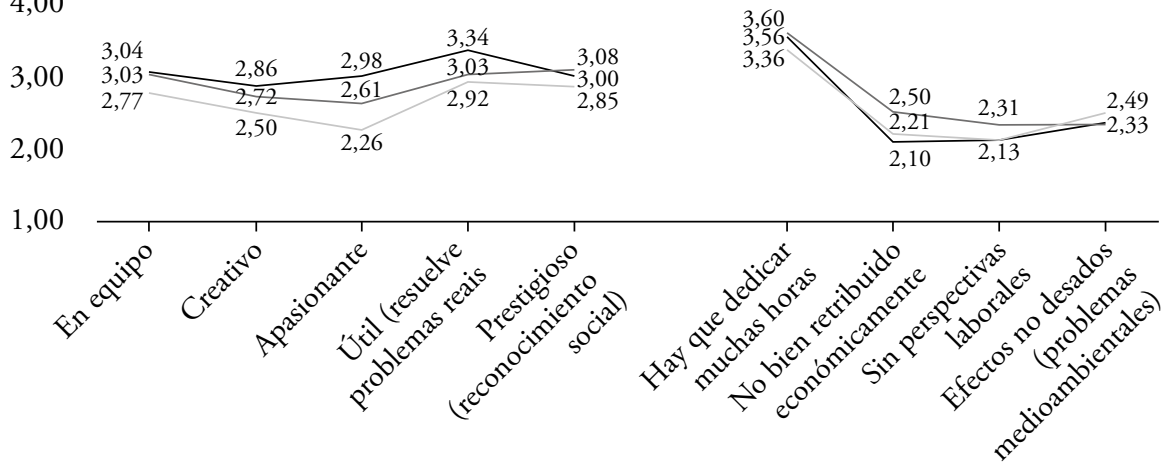

Relacionado $\quad$ No relacionado NS/NC 
En cuanto a la segunda hipótesis, respecto a la influencia de los factores académicos, y más concretamente sobre la figura del profesor, cuando se les preguntó si consideraban que uno de los inconvenientes para la elección vocacional podría ser que "los profesores no motivaban para realizar estudios superiores relacionados" los estudiantes que cursarán bachilleratos no relacionados (media = 3.05) manifestaban estar más de acuerdo (66,9\%), aunque no se hallaron diferencias estadísticamente significativas, mientras que solo el 43,4\% de los que cursarán un bachillerato relacionado no lo afirma (media $=2,87$ ), opinando que sus profesores sí les motivaron. Es decir, que la influencia del profesor parece tener mayor repercusión para los que no se sintieron atraídos por estas disciplinas, y eligieron cursar otro bachillerato no relacionado.

Sobre su opinión respecto a los contenidos de las materias, el 66,9\% de los alumnos que han elegido un bachillerato relacionado piensan que las asignaturas de Ciencias Naturales y Tecnología (cursadas en los tres años anteriores) les motivaron a proseguir sus estudios en esas áreas, hallándose diferencias estadísticamente significativas respecto a los alumnos que no cursarán bachilleratos relacionados $(\mathrm{F}=7.397, \mathrm{p}$ $=0.001)$. En concreto, de acuerdo con la Tabla 1 se observa que los estudiantes que van a cursar un bachillerato relacionado están más de acuerdo en que dichas materias son interesantes, útiles y favorecen el pensamiento crítico, según las diferencias estadísticamente halladas $(\mathrm{p}<0.05)$. Ambos grupos, sin existir diferencias destacables ( $p>0.05$ ), están en desacuerdo con otros aspectos motivacionales como la idea de que estas materias y sus contenidos puedan ser divertidos o desarrollar la creatividad.

En cuanto a las dificultades, y considerando que el desempeño de la competencia científica del alumnado en nuestro país aún se encuentra muy por debajo de los estándares de calidad que vienen señalando los marcos de evaluación de los organismos europeos e internacionales (OECD, 2013), llama la atención que los

\section{Tabla 1}

Media de grado de acuerdo (4) y desacuerdo (1) según la media, en los dos grupos y nivel de significación (Sig.).

\begin{tabular}{l|c|c|c|c}
\hline $\begin{array}{l}\text { Las materias y sus } \\
\text { contenidos... }\end{array}$ & $\begin{array}{c}\text { Bachillerato } \\
\text { RELACIONADO }\end{array}$ & $\begin{array}{c}\text { Bachillerato } \\
\text { NO RELACIONADO }\end{array}$ & T & Sig. \\
\hline Son interesantes & 3,09 & 2,70 & 7,397 &, $001^{*}$ \\
\hline Son divertidas & 2,50 & 2,38 & 2,043 &, 132 \\
\hline Son útiles & 3,33 & 2,96 & 6,605 &, $002^{*}$ \\
\hline Favorecen pensamiento crítico & 3,05 & 2,75 & 4,755 &, $009^{*}$ \\
\hline Desarrollan la creatividad & 2,42 & 2,40 &, 066 &, 936 \\
\hline Son difíciles de entender & 2,39 & 2,79 & 6,101 &, $003^{*}$ \\
\hline Exigen más trabajo y estudio & 3,68 & 3,17 & 6,997 &, $001^{*}$ \\
\hline
\end{tabular}


alumnos no estén de acuerdo en que las materias científico-tecnológicas sean "difíciles de entender", si bien, las diferencias significativas halladas entre los grupos confirmarían que los estudiantes que cursarán un bachillerato no relacionado están más de acuerdo. Y, en cuanto al grado de esfuerzo y exigencia, se encontró un elevado nivel de acuerdo, más concluyente entre los estudiantes que cursarán un bachillerato relacionado, de acuerdo con las diferencias estadísticamente significativas halladas.

Por último, se realizó un análisis de la metodología empleada por el profesor en las materias cursadas, observándose que las actividades consideradas como tradicionales (ver Figura 2) fueron las más frecuentes, especialmente la exposición por parte del profesor y el uso del libro de texto. Y, al contrario, las innovadoras (parte de arriba de la Figura 2), que se relacionan con la aplicación práctica del conocimiento científico (desarrollo de proyectos, debates o uso de noticias de prensa sobre resultados de investigaciones) o el conocimiento directo de la profesión, para incentivar su atractivo profesional (visitas a centros de investigación, museos, laboratorios...) fueron las menos frecuentes desde su opinión retrospectiva. Esto indica, como ha revelado también el estudio comparativo de Vázquez y Manassero (2015) que las experiencias escolares que pueden incidir en la elección vocacional siguen basándose en un modelo tradicional de enseñanza.

\section{Figura 2}

Actividades empleadas en la enseñanza/aprendizaje de las disciplinas científico-tecnológicas, más frecuentes (4) a menos frecuentes (1).

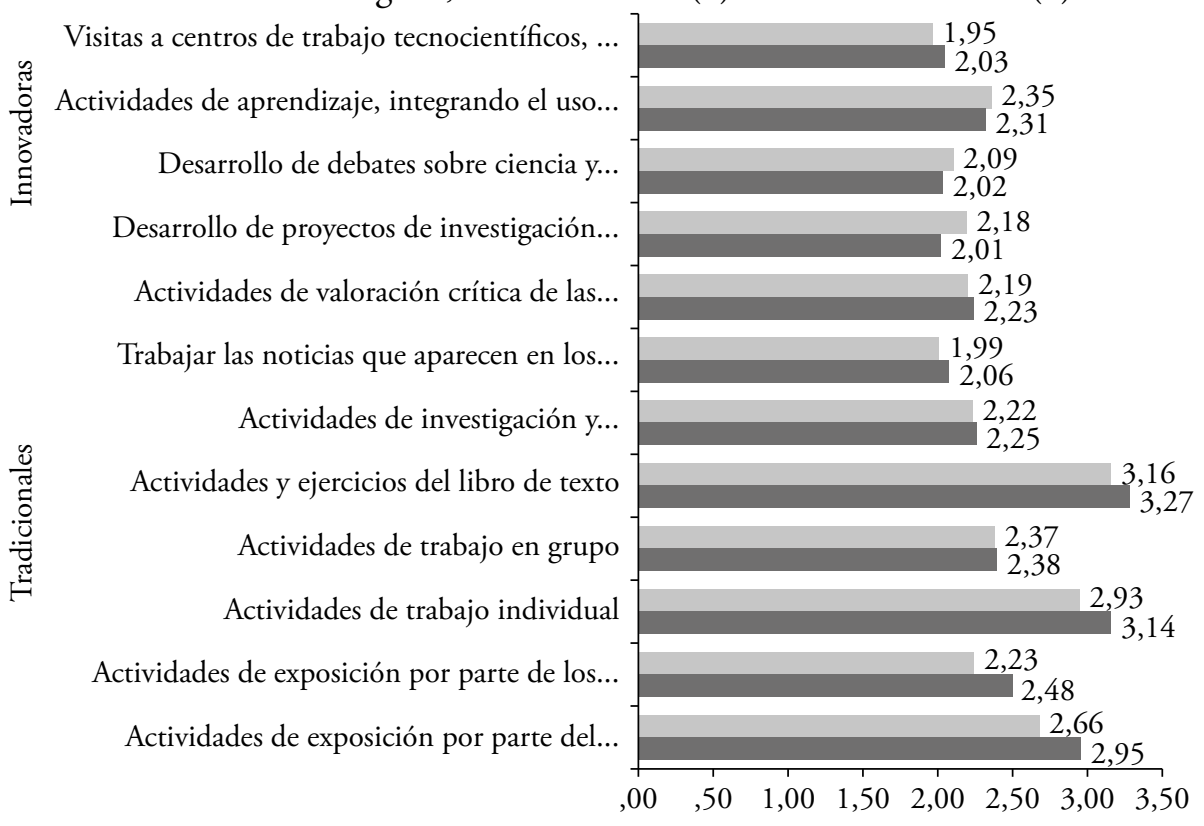

No relacionado

Relacionado 
Por todo ello, respecto a la segunda hipótesis planteada en cuanto a los factores académicos, los datos revelarían que efectivamente existe una percepción distinta de las materias, de su nivel de exigencia o del papel del profesor, entre los dos grupos, siendo más determinantes estos factores para los que no proseguirán estudios relacionados, manifestando que se sienten más desmotivados por la forma y la influencia de los profesores que tuvieron en los cursos anteriores. Sin embargo, los alumnos que cursarán bachilleratos relacionados han generado una percepción más positiva, y también realista - son más conscientes de que exigen más carga de trabajo - de lo que ha supuesto y supondrá estudiar estas materias. Teniendo en cuenta que sus juicios se basan en su experiencia retrospectiva de las materias obligatorias de la ESO, habiéndolas cursado con los mismos profesores y contenidos, son destacables las diferencias en las valoraciones de los dos grupos. Por ello, en aras a aumentar la vocación científico-tecnológica, se deberían considerar sus valoraciones, es decir, que para que los alumnos se motiven a estudiar bachilleratos superiores sigue siendo necesario hacer que estas materias y sus contenidos resulten interesantes y útiles, destacando también la importancia de desarrollar el pensamiento crítico, como competencia clave, la cual también les requerirá más tiempo de trabajo y estudio.

Finalmente, en cuanto a tercera hipótesis los alumnos opinaron que de todas las personas relacionadas con el ámbito científico-tecnológico que pueden tener una influencia en su elección vocacional, no son los familiares que trabajan en ese ámbito los que tienen importancia (media $=2.08)$, ni siquiera sus profesores de Ciencias Naturales o Tecnología (media $=2.33)$.

Sin embargo, sí están de acuerdo en que "no se conoce el trabajo que desempeñan este tipo de profesiones (científicas, tecnológicas)", tanto los estudiantes que cursarán bachilleratos relacionados (media $=3,06)$, como los que no (media $=3,08$ ).

También, consideran que "hay otras carreras profesionales con más salidas y expectativas laborales", siendo uno de los factores sociales donde se ha hallado un porcentaje de acuerdo más elevado entre los estudiantes (medias superiores a 3,5), sin hallarse diferencias estadísticamente significativas entre los grupos por modalidad de bachillerato. Dada su elevada valoración, consideramos que el conocimiento del mercado de trabajo y las salidas profesionales sí sería un factor vocacional decisivo para los alumnos.

Por último, en cuanto a los estereotipos de género, no se encontraron manifestaciones ni diferencias significativas que directamente indicaran que este fuera un factor de influencia, si bien, los que mayoritariamente eligieron cursar un bachillerato relacionado fueron hombres (61\%). Es destacable que las mujeres estuvieran más de acuerdo en la afirmación de que no se conoce la profesión (media $=3,2$ ), como se constata en otros estudios, donde se refiere a cierta presión cultural y a la existencia de menos modelos de mujeres científicas (OSBORNE; SIMON; COLLINS, 2003; VÁZQUEZ \& MANASSERO, 2015; SÁINZ \& MENESES, 2018).

En conclusión, los resultados han puesto de manifiesto que: por un lado, la elección vocacional hacia estudios superiores científico-tecnológicos no 
está fuertemente determinada por la tradición familiar, y sí por los factores académicos. Para los alumnos de nuestra muestra la tradición familiar no ha sido determinante ni en la elección, ni en la configuración de una visión positiva y realista de la profesión, como sí parecía ser relevantes en etapas pasadas (FERRY; FOUAD; SMITH, 2000; KELLER \& WHISTON, 2008).

Por otro lado, los estudiantes manifestaron que las metodologías tradicionales, predominantes en las materias de Ciencias Naturales y Tecnología de los cursos anteriores, influyeron en su elección vocacional, especialmente para los que no proseguirán estudios superiores relacionados, que no consideraron que estas materias fueran interesantes ni útiles, ni que sus profesores les motivaran a elegir estudios relacionados. Conclusiones que están relacionadas no tanto con investigaciones que han hablado del sentimiento de autoeficacia y la necesidad de invertir más esfuerzo para superarlas (BANDURA et al., 2001) sino más bien, con otras investigaciones donde se incide en las creencias de autoeficacia de los profesores (MCKINNON \& LAMBERTS, 2014), contribuyendo a crear una imagen generalizada de estas materias como más difíciles de superar.

$\mathrm{Y}$, en último término, con relación a los factores sociales, hemos comprobado que el imaginario social de la profesión científico que poseen los estudiantes de la ESO se configura a partir de la elección vocacional, encontrándose diferencias que indican que los estudiantes que deciden cursar un bachillerato científico o tecnológico, poseen una visión más positiva y realista de la profesión.

\section{Discusión, implicaciones y derivaciones}

Los resultados y las conclusiones resultantes, manifiestan, en términos generales, que existe un gran desconocimiento de la profesión, por lo que actividades prácticas, innovadoras y motivadoras, que conecten los centros educativos con los centros de investigación de su comunidad, o la visita en clase de un científico que les explique su profesión, podrían tener efectos positivos en su elección vocacional. La consideración global alude que quizás en ese imaginario social, las carreras científicas y las tecnológicas no tengan el mismo prestigio social (LOBERA \& TORRES, 2014), aunque en nuestro estudio solo analizó la visión del científico, con relación al Bachillerato elegido, conducente a carreras del ámbito científico. Sin embargo, es importante ampliar el impacto derivado de la integración de conocimientos esenciales del mundo científico y tecnológico, para desarrollar una visión positiva y conjunta del conocimiento científico-tecnológico. Para que la percepción del prestigio se equilibre, los estudiantes deberían aprender a compartir percepciones homogéneas de prestigio (WEGENER, 1992), así como procedimientos y habilidades que les conviertan en protagonistas de la difusión de la cultura tecnocientífica de su comunidad, lo que se traduciría en un cambio de perspectiva global con la que los alumnos, futuros ciudadanos, miran el mundo 
y generan propuestas de mejora, desde el valor que tiene el conocimiento y las profesiones que contribuyen a su generación e innovación.

En conjunto, estos resultados nos obligan a redefinir, nuevamente, los procesos de enseñanza y aprendizaje. Los rasgos de la sociedad del conocimiento marcan un saber cada vez más extenso, un conocimiento tendente a la fragmentación y un ritmo de producción de ese conocimiento que es cada vez más acelerado y, por tanto, su obsolescencia también crece. Rasgos que conducen a una nueva perspectiva del conocimiento que es la que marca procesos de enseńanza y aprendizaje abiertos, flexibles, planteando así una visión de conocimiento como proceso constructivo, hecho este que permite vincular la formación con la investigación, la reflexión, la creatividad, el espíritu emprendedor, el reclamado empoderamiento del alumno y el fomento de la auto-responsabilidad social y académica, equilibrando pensamiento, lenguaje y comportamiento.

El cuestionamiento del modelo educativo desde la cultura predominante basada en la lógica tradicional de las disciplinas, a un modelo formativo que reintegre la visión académica, la profesional y la vital está expuesto en base a resultados como los que presentamos. Hemos de plantear, o al menos discutir pedagógicamente si el modelo organizativo de las enseñanzas conducentes a un título, acumulativo y fraccionado, es suficiente o se podría optimizar desde un modelo integrador y constructivo. Se trataría de buscar espacios curriculares de integración y metodologías de aprendizaje y enseńanza que propicien un acercamiento a la realidad profesional, también la científica, como vía para conseguir un aprendizaje significativo, profundo y constructivo (PEDRINACI et al., 2012).

Un debate que para muchos resulta atávico, pero que en numerosas ocasiones está presente en las aulas abriendo, a su vez, otro debate: el de formar al profesorado para situarlo en las condiciones óptimas de llevar a cabo el demandado cambio en la cultura del aprendizaje. No siempre la formación del profesorado se corresponde con lo que demanda el mundo del conocimiento (CONTRERAS, 2016; MEDEIROS et al., 2016). El problema estriba en la descontextualización de la formación. Existe un imaginario previo en torno a la figura clásica del profesor que guía el diseño de los procesos formativos en base a imperativos resolutivos, reduccionistas y excesivamente didactistas, que simplifican la lógica formativa, reduciendo la formación a mero control de variables y aplicación de técnicas y métodos.

Los argumentos expuestos con anterioridad invitan a una formación inicial del profesorado más creativa y creadora, superando idolatrías metodológicas, fomentando la iniciativa del futuro profesor desde rutas formativas flexibles, polivalentes, inacabadas. Idéntico recorrido que debe seguir igualmente la formación continua, superando prácticas fundamentadas desde cursos didáctico-pedagógicos-políticos generalizados, amparados en lo política y económicamente correcto, al margen de una responsabilidad social compartida y de una cooperación interinstitucional e internacional, concibiéndose su formación no como una obligación sino como parte de su desarrollo vital-profesional. Estamos convencidos de 
que esto supondrá esfuerzos también más allá del profesorado, que impliquen de manera colaborativa a todas las instancias educativas, donde la educación social y no-formal tiene un rol fundamental (SANTILLÁN et al., 2017).

Y, por último, en cuanto a la difusión del conocimiento científico que contribuye a la construcción del imaginario social de la profesión, resulta cada vez más necesario desarrollar nuevas alternativas donde se conecte el conocimiento curricular con la aplicación práctica, y los últimos avances que se publican en tiempo real y contribuyen a solucionar problemáticas cotidianas. En la enseñanza superior ya se aplican modelos curriculares innovadores (Universidad de MacMaster, o Team Academy de Finlandia, entre otros), que pueden ser inspiradoras para la enseñanza media, que se caracterizan por ser modelos híbridos (FERNÁNDEZ et al., 2013) combinan el aprendizaje curricular más estático, con la resolución de problemas, casos y proyectos prácticos, cuyo objetivo es incrementar el aprendizaje activo y disminuir las experiencias de aprendizaje pasivo, para que los alumnos despierte su interés por la creación del conocimiento científico.

\section{Referencias}

ACEVEDO-DÍAZ, J. A.; GARCÍA-CARMONA, A. Algo antiguo, algo nuevo, algo prestado". Tendencias sobre la naturaleza de la ciencia en la educación científica. Revista Eureka sobre Enseńanza y Divulgación de las Ciencias, v. 13, n. 1, p. 3-19, 2016.

ALBORNOZ, M. Ciencia y Tecnología para el desarrollo. Revista Internacional de Investigación en Ciencias Sociales, v. 11, n. 2, p. 161-163, 2015. http://dx.doi. org/10.18004/riics.2015.diciembre.161-163

ALCÍBAR, M. Comunicación pública de la ciencia y la tecnología: una aproximación crítica a su historia conceptual. Arbor, v. 191, n. 773, 2015. https://doi.org/10.3989/ arbor.2015.773n 3012

ALONSO, J.; RAMÓN, J. Prácticas educativas familiares y autoestima. Psicothema, v. 17, n. 1, p. 76-82, 2005.

ANGLADA, L. M.; ABADAL, E. ¿Qué es la ciencia abierta? Anuario ThinkEPI, v. 12, p. 292-298, 2018. https://doi.org/10.3145/thinkepi.2018.43

ARCHER, L.; DEWITT, J.; OSBORNE, J.; DILLON, J.; WILLS, B.; WONG, B. Science aspirations, capital, and family habitus how families shape children's engagement and identification with science. American Educational Research Journal, v. 49, n. 5, p. 881-908, 2012. https://doi.org/10.3102\%2F0002831211433290

BANDURA, A.; BARBARANELLI, C.; CAPRARA, G. V.; PASTORELLI, C. Self-efficacy beliefs as shapers of children's aspirations and career trajectories. Child Development, v. 72, n. 1, p. 187-206, 2001.

BEAL, S. J.; CROCKETT, L. J. Adolescents' occupational and educational aspirations and expectations: Links to high school activities and adult educational attainment. Developmental Psychology, v. 46, n. 1, p. 258-265, 2010. https://doi.org/10.1037/a0017416 
BECKER, F. S. Why not opt for a career in Science and Technology? An analysis of potentially valid reasons. In: ANNUAL CONFERENCE OF SEFI. ATTRACTING YOUNG PEOPLE TO ENGINEERING. ENGINEERING IS FUN, 37., 2009. Anales... 2009.

BERENDS, M. Survey methods in educational research. In: GREEN, J. L.; CAMILA, G.; ELMORE, P. B. (orgs.). Handbook of complementary methods in education research. Washington, D.C.: American Educational Research Association, 2006. p. 623-640.

BORGES, I. M.; PIRES, D. M.; DELGADO, J. ¿Qué mejoras se han alcanzado respecto a la Educación Científica desde el enfoque Ciencia-Tecnología-Sociedad-Ambiente en el nuevo Currículo Oficial de la LOMCE de $5^{\circ}$ y $6^{\circ}$ curso de Primaria en España? Revista Eureka sobre Enseńanza y Divulgación de las Ciencias, v. 15, n. 1, p. 1101, 2018. https://doi.org/10.25267/Rev_Eureka ensen divulg_cienc.2018.v15.i1.1101

BRONFENBRENNER, U. Making Human Beings Human. Bioecoligal perspectives on human development. Thosand Oaks: Sage, 2005.

CONTRERAS, S. Pensamiento pedagógico en la enseñanza de las ciencias. Análisis de las creencias curriculares y sus implicaciones para la formación de profesores de enseñanza media. Formación Universitaria, v. 9, n. 1, p. 15-24, 2016. http://dx.doi.org/10.4067/ $\underline{\text { S0718-50062016000100003 }}$

CORTÉS, P. A.; CONCHADO, A. Los contextos parentales y académicos y los valores laborales en la toma de decisiones en Bachiderato. Estudios sobre Educación, v. 22, p. 93-114, 2012.

DEWITT, J.; ARCHER, L. Who Aspires to a Science Career? A comparison of survey responses from primary and secondary school students. International Journal of Science Education, v. 37, n. 13, p. 2170-2192, 2015. https://doi.org/10.1080/09500693.2015.1071899

DEWITT, J.; ARCHER, L.; OSBORNE, J. Science-related aspirations across the primary-secondary divide: Evidence from two surveys in England. International Journal of Science Education, v. 36, n. 10, p. 1609-1629, 2014. https://doi.org/10.1080/09500 $\underline{693.2013 .871659}$

EUROPEAN COMMISSION. Science Education Now: A Renewed Pedagogy for the Future or Europe. Buselles: European Commission, Community Research, 2007.

FECYT. Percepción social de la ciencia y la tecnología. Madrid: MEC, 2015.

FERNÁNDEZ, I.; GUISASOLA, G.; GARMENDIA, M.; ALKORTA, I.; MADINABEITIA, A. ¿Puede la formación tener efectos globales en la universidad? Desarrollo docente, metodologías activas y curriculum híbrido. Infancia y Aprendizaje: Journal for the Study of Education and Development, v. 36, n. 3, p. 387-400, 2013. https://doi.org/10.1174/021037013807532990

FERRY, T. R.; FOUAD, N. A.; SMITH, P. L. The role of family context in a social cognitive model for career-related choice behavior: A math and science perspective. Journal of Vocational Behavior, v. 57, n. 3, p. 348-364, 2000. http://dx.doi.org/10.1006/ jvbe. 1999.1743

FURTAK, E. M.; SEIDEL, T.; IVERSON, H.; BRIGGS, D. C. Experimental and quasiexperimental studies of inquiry-based science teaching. Review of Educational Research, v. 82, n. 3, p. 300-329, 2012. https://doi.org/10.3102\%2F0034654312457206 
GARCÍA, M. E.; GUTIÉRREZ, A. B. B.; HERRERO, E. T.; MENÉNDEZ, R. C.; PÉREZ, J. C. N. El contexto sí importa: identificación de relaciones entre el abandono de titulación y variables contextuales. European Journal of Education and Psychology, v. 9, n. 2, p. 79-88, 2016. https://doi.org/10.1016/j.ejeps.2015.06.001

HERNÁNDEZ, M. P. S. Déficit vocacional en la era digital. Tendencias Sociales. Revista de Sociología, v. 1, n. 1, p. 197-224, 2018. https://doi.org/10.5944/ts.1.2018.21365

JANSEN, M.; SCHERER, R.; SCHROEDERS, U. Students' self-concept and self-efficacy in the sciences: Differential relations to antecedents and educational outcomes. Contemporary Educational Psychology, v. 41, p. 13-24, 2015. https:// psycnet.apa.org/doi/10.1016/j.cedpsych.2014.11.002

KELLER, B. K.; WHISTON, S. C. The role of parental influences on young adolescents' career development. Journal of Career Assessment, v. 16, n. 2, p. 198-217, 2008. https:// doi.org/10.1177\%2F1069072707313206

KERLINGER, F; LEE, H. Investigación del comportamiento, métodos de investigación en ciencias de la educación. México: McGraw Hill, 2002.

$\mathrm{KO}, \mathrm{H}$. In science communication, why does the idea of a public deficit always return? How do the shifting information flows in healthcare affect the deficit model of science communication? Public Understanding of Science, v. 25, n. 4, p. 427-432, 2016. https://doi.org/10.1177\%2F0963662516629746

KRAPP, A.; PRENZEL, M. Research on interest in science: Theories, methods, and findings. International Journal of Science Education, v. 33, n. 1, p. 27-50, 2011. https://doi.org/10.1080/09500693.2010.518645

LOBERA, J.; TORRES, C. T. El prestigio social de las profesiones tecnocientíficas. Percepción social de la ciencia y la tecnología. España: FECYT, 2014.

MARTÍNEZ, A.; ZURITA, F. El rol que tiene la influencia familiar y su nivel académico, en los itinerarios curriculares de estudiantes de último curso. Educatio Siglo XXI, v. 32, n. 2, p. 267-286, 2014. https://doi.org/10.6018/j/202251

MCKINNON, M.; LAMBERTS, R. Influencing science teaching self-efficacy beliefs of primary school teachers: A longitudinal case study. International Journal of Science Education, Part B, v. 4, n. 2, p. 172-194, 2014. https://doi.org/10.1080/21548455.2013.793432

MEDEIROS, B.; MONTANARI, P.; CASSIANI, S.; VON LINSINGEN, I. Cuestiones sociotecnológicas en la formación de profesores de ciencias: una perspectiva discursiva. Revista Iberoamericana de Educación, v. 70, n. 1, p. 115-128, 2016. https://doi. org/10.35362/rie70176

NAVAL, C.; ARBÚES, E. Del uso de Internet en la promoción de virtudes sociales. Un caso concreto: Parlamento cívico. Teoría de la Educación. Revista Interuniversitaria, v. 27, n. 1, p. 33-52, 2015. http://dx.doi.org/10.14201/teoredu20152713352

ORGANIZACIÓN PARALACOOPERACIÓNYELDESARROLLOECONÓMICOS (OECD). PISA 2015. Draft Science Framework. Paris: OECD, 2013.

OSBORNE, J.; DILLON, J.; COLLEGE, K. Science education in Europe: Critical reflections. A report to the Nuffield Foundation. Londres: King's College, 2008. 
OSBORNE, J.; SIMON, S.; COLLINS, S. Attitudes towards science: A review of the literature and its implications. International journal of science education, v. 25, n. 9, p. 1049-1079, 2003.

OSPINA, D. L. P. Jóvenes Nini, ¿limitante para la ciencia, la tecnología y la innovación en Colombia? AD-minister, n. 32, p. 83-106, 2018. http://dx.doi.org/10.17230/ad-minister.32.4 PEDRINACI, E.; CAAMAÑO, A.; DE LEÓN, P. C.; PRO BUENO, A. Once ideas clave. El desarrollo de la competencia científica. Barcelona: Graó, 2012.

PEÑA, J. V.; INDA, M.; RODRÍGUEZ, C.; FERNÁNDEZ, C.-M. Perceived Supports and Barriers for Career Development for Second-Year STEM Students. Journal of Engineering Education, v. 105, n. 2, p. 341-365, 2016. https://doi.org/10.1002/jee.20115

PÉREZ, G. El conocimiento científico y sus carcomas. Teoría de la Educación. Revista Interuniversitaria, v. 23, n. 2, p. 19-43, 2011. http://dx.doi.org/10.14201/ted.8644

PLAZA, M. B. Fomento del interés por la ciencia y la tecnología para estudiantes de enseńanza secundaria. Tese (Doctorado en Educación) - Universidad Politécnica de Madrid, Madrid, 2015.

POLINO, C.; CHIAPPE, M. Proyecto "Percepción de los jóvenes sobre la ciencia y la profesión científica”. Encuesta en Buenos Aires. Reporte final. Buenos Aires: Centro de Altos Estudios Universitarios, Organización de Estados Iberoamericanos (OEI), Observatorio de la Ciencia, la Tecnología y la Innovación, 2011.

POTVIN, P.; HASNI, A. Interest, motivation and attitude towards science and technology at K-12 levels: a systematic review of 12 years of educational research. Studies in Science Education, v. 50, n. 1, p. 85-129, 2014. https://doi.org/10.1080/03057267.2014.881626

ROMERO, F. E. C.; ESPINOZA-ROMO, A. V.; VALADEZ, A. A. V.; CAMPOS, T. G.; GARCIA, L. ¿Cómo valoran los adolescentes a la ciencia y a los científicos? Análisis desde una aproximación metodológica mixta. Caleidoscopio, v. 21, n. 37, p. 55-74, 2018. https://doi.org/10.33064/37crscsh902

RUÍZ-CARRASCOSA, J.; MOLERO, D. Ocupación laboral y ocupación complementaria de los titulados universitarios. Bordón, v. 62, n. 1, p. 109-122, 2010.

SÁINZ, M.; MENESES, J. Brecha y sesgos de género en la elección de estudios y profesiones en la educación secundaria. Panorama Social, v. 27, p. 23-31, 2018.

SANTILlÁN, J. E.; COLOMBO, E. M.; JARMA, N.; PÉREZ, A. I.; ISGRO, C. A.; CHIRRE, A.; LAZARTE, E. L. Preguntas desde el interior: los jóvenes, la ciencia y la tecnología. Revista Iberoamericana de Educación, v. 75, n. 2, p. 21-40, 2017. https:// doi.org/10.35362/rie7522630

STEKOLSCHIK, G., DRAGHI, C.; ADASZKO, D.; GALLARDO, S. Does the public communication of science influence scientific vocation? Results of a national survey. Public Understanding of Science, v. 19, n. 5, p. 625-637, 2010. https://doi. org/10.1177/0963662509335458

TASKINEN, P. H.; DIETRICH, J.; KRACKE, B. The Role of Parental Values and Childspecific Expectations in the Science Motivation and Achievement of Adolescent Girls and Boys. International Journal of Gender, Science and Technology, v. 8, n. 1, p. 103-123, 2015. 
TASKINEN, P. H.; SCHÜTTE, K.; PRENZEL, M. Adolescents' motivation to select an academic science-related career: the role of school factors, individual interest, and science self-concept. Educational Research and Evaluation, v. 19, n. 8, p. 717-733, 2013. https://doi.org/10.1080/13803611.2013.853620

THOMAS, R.; WHYBROW, K.; SCHARBER, C. A conceptual exploration and participation. Section I: Introduction and early perspectives. Educational Philosophy and Theory, v. 44, n. 6, p. 594-613, 2012. https://doi.org/10.1111/j.1469-5812.2010.00731.x

VÁZQUEZ, A.; MANASSERO, M. A. Factores actitudinales determinantes de la vocación científica y tecnológica en secundaria. Cultura y Educación, v. 21, n. 3, p. 319-330, 2009. https://doi.org/10.1174/113564009789052280

VÁZQUEZ, Á.; MANASSERO, M. A. La elección de estudios superiores científicotécnicos: análisis de algunos factores determinantes en seis países. Revista Eureka sobre Enseñanza y Divulgación de las Ciencias, v. 12, n. 2, p. 264-277, 2015.

VILACA, M. M. Mala conducta científica: un enfoque comparativo y crítico para informar una reflexión sobre el tema. Revista Brasileira de Educaçáo, v. 20, n. 60, p. 245-269, 2015. http://dx.doi.org/10.1590/S1413-24782015206012

WEGENER, B. Concepts and measurement of prestige. Annual Review of Sociology, v. 18, n. 1, p. 253-280, 1992. https://doi.org/10.1146/annurev.so.18.080192.001345

\section{SOBRE LOS AUTORES}

José Manuel Muñoz Rodríguez es Dr. en Pedagogía. Profesor Titular de Universidad. Universidad de Salamanca.

María José Hernández Serrano es Dra. en Pedagogía. Profesora Contratado Doctor. Universidad de Salamanca.

Sara Serrate González es Dra. en Pedagogía. Profesora Ayudante Doctor. Universidad de Salamanca.

Recibido en el 29 de octubre de 2017.

Acepto en el 27 de febrero de 2019.

(C) 2019 Centro de Estudos Educação e Sociedade - CEDES Este é um artigo de acesso aberto distribuído nos termos de licença Creative Commons. 